\title{
КОНТУР ЧИСТО ЭЛЕКТРОННОЙ ЛИНИИ В ЗАВИСЯЩИХ ОТ ВРЕМЕНИ СПЕКТРАХ: СУЖЕНИЕ И ОСЦИЛЛЯЦИИ
}

INNA REBANE, A. TUUL. PUHTELEKTRONJOONE KONTUUR AJAST SOLTUVATES SPEKTRITES: KITSENEMINE JA OSTSILLATSIOONID

INNA REBANE, A, TUUL. THE CONTOUR OF PURE-ELECTRONIC LINE IN TRANSIENT RESONANCE SECONDARY RADIATION SPECTRA: NARROWING AND OSCILLATIONS

(Представлена К. К. Ребане)

В ['] были приведены формулы зависящего от времени спектра резонансного вторичного свечения (РВС) в модели с одним локальным колебанием, а также некоторые результаты выполненных по ним машинных расчетов. Рассмотрение в [ $\left.{ }^{1}\right]$ было проведено для переходного спектра $I(\Omega, t)$ в предельном случае бесконечно точного спектрального разрешения $\eta=0$ (см. $\left.\left[{ }^{2}\right]\right)$, т. е. была найдена вероятность $W(\Omega, t)$ того, что к моменту времени $t$ будет испущен фотон частоты $\Omega(t=$ $=0$ - момент, когда максимум возбуждающего светового импульса проходит через примесной центр). В частности, было показано, что чисто электронная линия (ЧЭЛ) люминесценции, излученная ко времсни $t$, где $t \approx \tau_{\text {rel }} \ll \tau_{\text {opt }}$, является «горячей», т. е. как по интенсивности, так и по контуру (ширине) она в общем такая же, как все остальные линии люминесценции, в том числе линии горячей люминесценции.

C увеличением $t$ ЧЭЛ начинает «охлаждаться»: наряду с последовательным ростом интегральной интенсивности происходит ее сужение, которое сопровождается осцилляциями на крыльях ее контура (см. [ ${ }^{1}$ ] рис. 2). Только при $t>\tau_{\text {opt }}$ ЧЭЛ становится хорошо известной, весьма узкой лоренцевой линией спектра люминесценции примесного центра, измеренной в стационарных условиях (см. $\left.\left[{ }^{3}\right]\right)$.

Цель настоящего сообщения - привести результаты машинных расчетов, иллюстрирующие сужение и осцилляции на крыльях ЧЭЛ в процессе ее «охлаждения». Расчеты выполнены по формулам и программам, в которые введено и спектральное разрешение $\eta \neq 0$ по методу, предложенному в $\left[{ }^{2}\right]$. ЧЭЛ.

Из рис. 1 ясно видно временное развитие осцилляций на крыльях

1. Отношение высоты первого побочного максимума (перед окончательным исчезновением структуры - ближайшей точки перегиба) к высоте центрального пика равняется примерно $3-5 \%$.

2. Удвоение времени $t$ приводит к уменьшению расстояния между соседними максимумом и минимумом примерно в два раза. В этом кроется первая причина исчезновения структуры при больших $t$ : она 

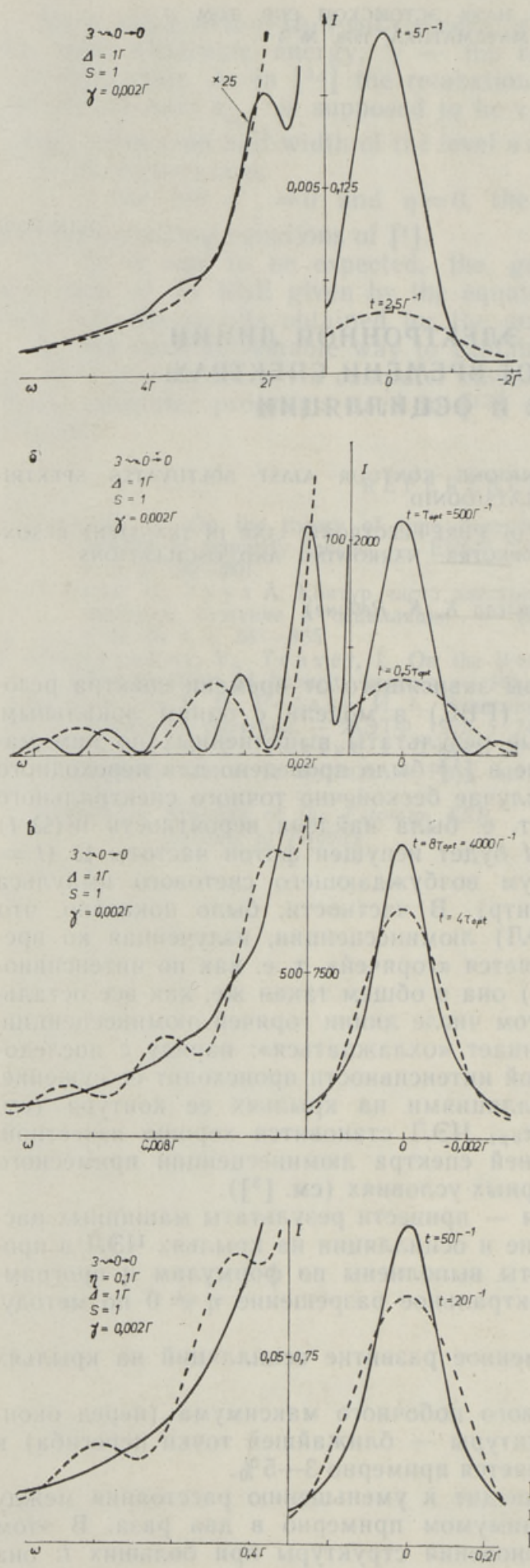

Рис. 1. Развитие во времени вероятности $(W(\Omega, t)=I(\Omega, t ; \eta)$ при $\eta=0$ ) чисто электронной линии при возбуждении на $n=3$ колебательный уровень. Стоксовы потери $S=1$; полуширина возбуждаюшего пакета $\Delta=1 \Gamma ; \quad \tau_{o p t} t^{-1} \equiv \gamma=$ $=0,002 \Gamma$, где $\Gamma$ релаксационная полуширина уровня $n=1$.

становится для заданной конечной величины спектрального разрешения $\eta$ неразличимой (см. рис. 2).

3. Начиная с некоторого достаточно большого $t(t>$ $\left.>\tau_{o p t}\right)$ относительная глубина модуляции контура убывает. В этом видится вторая (и определяющая) причина исчезновения осциллирующей структуры при $t \gg \tau_{\text {opt }}$.

Вполне очевидно (соответствующие количественные соотношения даются формулами зависящих от времени полуширин линий спектров РВС), что если промежуток измерения выбрать достаточно длинным (больше $\left.\tau_{o p t}\right)$ и так, чтобы он начинался сразу после «горячего» перехода становления ЧЭЛ, то ширина полученной линии будет меньше естественной ширины, определяемой $\tau_{\text {opt }}$.

Әкспериментальное изучение описанного поведения ЧЭЛ в оптических спектрах примесей в кристаллах чрезвычайно затруднено из-за сильного неоднородного уширения этой линии (см. $\left.\left[{ }^{3}\right]\right)$. Часто оно достигает одного $C M^{-1}$ или даже больше, т. е. становится примерно равным величине релаксационного уширения Г. Зна-

Рис. 2. Спектр $I(\Omega, t ; \eta)$ при временах $t=20$ и $50 \quad \Gamma^{-1}$. Спектральное разрешение $\eta=0,1 \Gamma$; остальные параметры те же, что и на рис. 1. 
чит, оно не сможет существенно мешать измерениям осцилляций только у совсем еще «горячих» ЧЭЛ, когда ширина центрального пика и расстояние между соседними максимумами и минимумами еще порядка $\Gamma$, т. е. при временах порядка $t=5 \Gamma^{-1} \approx 10^{-11}$ сек (ср. с рис. $\left.1, a\right)$. Неоднородное уширение в эффекте Мёссбауэра намного меньше (см. $\left[{ }^{2}\right]$. Поэтому как осцилляции на крыльях, так и сужение с ростом $t$ впервые экспериментально наблюдались у зависящих от времены мёссбауэровских спектров на примесных ядрах ${ }^{57} \mathrm{Fe}\left[{ }^{4}\right]$.

\section{Л И Т Е Р А Т У Р А}

1. Rebane, I. On the theory of time dependence of resonant secondary emission spectra of impurity centres. - ENSV TA Toimet., Füüs. Matem., 1978, v. 27, N 2, p. 192-201.

2. Хи жня ков В. В., Р еб ане И. К. Зависящие от времени спектры резонансного вторичного свечения. - ЖЭТФ, 1978, т. 74 , вып. 3, с. 885-896. H i zhn y a $\mathrm{kov}, \mathrm{V}$. On the theory of transient resonance secondary radiation spectra. Techn. Rep. of ISSP, Ser. A, N 860, Dec. 1977.

3. Р е б а н К. К. Элементарная теория колебательной структуры спектров примесных центров кристаллов. М., 1968.

4. Kobay a shi, T., Shimizu, S. Time-filtering effect in the Mössbauer spectrum. Phys. Letters, 1975 , v. $54 \mathrm{~A}, \mathrm{~N} 4$, p. $3: 1-312$. Re a lo, E., Ko ch, R. Timeresolved Mössbauer spectroscopy of ZeS.57Co crystal. - In: Abstr. of XX Congr. AMPERE, Tallinn, Aug. 1978.

\section{Институт физики \\ Академии наук Эстонской ССР}

Поступила в редакцию 21/VIII 1978 